\title{
Transtornos depressivos em crianças com leucemia linfoide aguda e com insuficiência renal crônica terminal / estudo de série de casos
}

\author{
Depressive disease in children with acute lymphocytic leukemia
}

and end stage of renal disease/case series study

Juliana Oliveira', José Marcelino Bandim², José Eulálio Cabral Filho³

\section{RESUMO}

Objetivo: Investigar a presença de transtornos depressivos em crianças portadoras de leucemia linfoide aguda (LLA) e insuficiência renal crônica terminal (IRCT) atendidas no IMIP. Método: Estudo descritivo do tipo série de casos, composto por 52 crianças entre 8 e 15 anos portadoras de LLA e de IRCT. Resultados: Três (5,8\%) casos preenchiam os critérios para episódio depressivo maior (EDM), sendo dois portadores de IRCT e um portador de LLA. Oito $(15,4 \%)$ preenchiam os critérios para transtorno distímico (TD), todos eles portadores de IRCT. A associação entre faixa etária e EDM não foi significativa ( $p=0,327)$. Entretanto, a faixa etária foi significante em relação ao TD ( $p=0,014)$, todos os seus portadores tinham entre 12 e 15 anos de idade. A associação entre os transtornos depressivos e o tempo de evolução da doença de base não foi significante. Contudo, observou-se uma tendência a quanto maior o tempo de evolução da doença de base, maior a associação com o TD. Conclusão: A frequência de EDM ficou dentro da faixa encontrada na literatura para escolares saudáveis, entretanto, a de TD foi mais alta. Não foram encontradas diferenças significantes entre as faixas etárias no diagnóstico de EDM. Porém, corroborando a literatura, a faixa etária maior prevaleceu em relação ao TD.

\section{ABSTRACT}

Objective: To Investigate the presence of depressive disease in children with acute lymphocytic leukemia (ALL) and end stage of renal disease (ESRD). Method: A case series study including 52 children suffering of ALL or ESRD aged 8 to 15 years. Results: Three patients (5.8\%) had major depressive episode (MDE), two of them with ESRD and one with ALL. Eight patients (15.4\%) had dysthymic disorder (DD), all of them had ESRD. The association between age and MDE was not meaningful. On other hand, the association between age and DD was significant, and all of the patients aged between 12 and 15 years. The association between depressive diseases and evolu-

\section{Keywords}

Depression, depressives symptoms, acute lymphocytic leukemia, end stage of renal disease.

Recebido em
6/3/2009
Aprovado em
30/7/2009

1 Instituto Materno-Infantil Prof. Fernando Figueira (IMIP), Recife, PE. 2 Universidade Autônoma de Barcelona (UAB), Espanha, Departamento de Psiquiatria. 3 Instituto de Medicina Integral Prof. Fernando Figueira - IMIP.

Endereço para correspondência: Juliana Oliveira Rua Antônio de Castro, 175, apto. 1302, Casa Amarela - 52070-080 - Recife, PE E-mail: julianaocc@gmail.com 


\section{INTRODUÇÃO}

Dentre as pesquisas sobre psicopatologia na infância e adolescência, têm-se destacado estudos sobre depressão (na sua forma sindrômica ou puramente sintomatológica). Tais estudos não só focalizam populações de forma geral, mas também populações específicas, como crianças com outras doenças pediátricas, tanto crônicas como agudas, associadas $^{1-8}$.

Os estudos pioneiros apontavam para uma maior prevalência de transtornos depressivos ou sintomatologia depressiva em crianças com câncer do que em populações de crianças sem doençâ, ${ }^{1,2}$. Entretanto, investigações mais recentes não têm reproduzido esses achados ${ }^{4-8}$. É provável que os métodos diagnósticos utilizados sejam responsáveis por essas diferenças, já que os primeiros estudos não utilizavam instrumentos estruturados, com critérios diagnósticos bem definidos, além de coexistirem com a ausência completa de instrumentos específicos para avaliar a sintomatologia depressiva em crianças e adolescentes ${ }^{1,2}$.

Alguns autores discutem a necessidade da utilização tanto de entrevistas semiestruturadas como de inventários para esse tipo de população. Argumentam em favor da elaboração/utilização de instrumentos mais específicos para essa população, pois, muitos itens dos instrumentos utilizados no estudo para avaliar a depressão podem ter seus escores alterados tanto pela sintomatologia clínica da doença de base, como pela influência dos efeitos colaterais dos tratamentos específicos?.

Uma criança diagnosticada com doença grave sofre a quebra de sua rotina e afazeres, a interrupção das atividades escolares e lúdicas, a separação de pessoas próximas, tanto pelas limitações que a doença provoca como pelos procedimentos hospitalares, forçando-a a se readaptar à nova forma de vida, com novas responsabilidades, hospitalizações, procedimentos dolorosos e modificação do seu corpo ${ }^{10-13}$.

O impacto da doença crônica na infância e adolescência tem sido reconhecido; e há evidências que indicam que o risco de problemas psicológicos é aproximadamente duas vezes maior, se comparado ao de crianças sem doença crônical ${ }^{14,15}$.

A associação entre depressão e doença crônica pode levar tanto a uma piora da evolução do quadro psiquiátrico como da doença de base, com menor aderência às rotinas terapêuticas, aumento da percepção de sintomas físicos inexplicáveis, além de maior morbidade e mortalidade. A depressão propriamente dita muitas vezes é subdiagnosticada e subtratada, especialmente, porque sintomas depressivos também podem ocorrer em doenças crônicas. A existência dessas associações indica a necessidade de uma avaliação adequada dos sintomas depressivos, que podem estar superpostos aos sintomas da patologia clínica ${ }^{16}$.
A dificuldade em se avaliar sintomas depressivos e definir o diagnóstico de depressão em pacientes clínicos gerou a necessidade de se estabelecer critérios que classificassem a questão, apesar de não haver consenso na literatura. Esses critérios podem ser agrupados em tipos de abordagens: a inclusiva, a excludente, a etiológica e a substitutiva. A meIhor abordagem para a prática clínica diária é a inclusiva, em que os sintomas depressivos devem ser contabilizados, independentemente de poderem ser explicados pela patologia clínica. Essa abordagem gera mais diagnósticos falsopositivos, porém diminui o risco de não se diagnosticar um quadro depressivo oligossintomático. A abordagem excludente retira os sintomas explicáveis pela patologia clínica, mas os critérios são muito variáveis de autor para autor, e por serem muito restritivos, acabam gerando muitos diagnósticos falso-negativos.

A abordagem etiológica é aquela em que o diagnóstico de depressão é definido a partir da inferência de que a patologia clínica causou o quadro depressivo, o que gera diagnóstico de baixa confiabilidade. Por último, a proposta de substituir um sintoma físico por um comportamental ou cognitivo, por exemplo, trocar alterações do sono por isolamento social, pode gerar multiplicidade de diagnósticos ${ }^{16}$.

Dessa forma, o critério de avaliação utilizado neste estudo foi o inclusivo, em que o objetivo principal foi investigar a ocorrência de episódio depressivo maior e de transtorno distímico, e suas diferenças na apresentação clínica em crianças com leucemia linfoide aguda (LLA) e insuficiência renal crônica terminal (IRCT) atendidas nos serviços de oncologia e nefrologia Pediátrica do IMIP.

\section{MÉTODO}

Trata-se de um estudo descritivo do tipo série de casos, com pacientes portadores de LLA ou IRCT, atendidos nos serviços de oncologia e nefrologia do Instituto Materno-Infanti Prof. Fernando Figueira (IMIP), no ano de 2006. O IMIP é um centro credenciado pelo Ministério da Saúde para transplantes renais e tratamento oncológico na cidade do Recife, que atende a pessoas de baixa renda, procedentes tanto da região metropolitana do Recife quanto do interior do estado. Participaram do estudo 52 pacientes entre 8 e 15 anos de idade, de ambos os sexos, sendo 27 portadores de IRCT e 25 portadores de LLA, aqui denominadas de doença de base. Foram excluídas: crianças que apresentavam comprometimento cognitivo, auditivo ou verbal suficientemente grave que impossibilitasse o entendimento do instrumento de avaliação utilizado no estudo; crianças portadoras de outros tipos de neoplasias ou doenças crônicas, que não a IRCT ou a LLA; crianças que tinham sido submetidas a radioterapia intracraniana; e aquelas que no momento da 
pesquisa não apresentavam condições clínicas para serem examinadas.

\section{Coleta de dados}

Os pacientes diagnosticados com LLA que participaram do estudo foram aqueles que estavam em tratamento quimioterápico. Os participantes com IRCT foram os pacientes que estavam sendo submetidos a hemodiálise, a diálise ou haviam feito transplante renal no serviço.

As crianças e seus pais ou responsáveis foram entrevistados nos setores de nefrologia ou de oncologia do IMIP, nos horários de atendimento preestabelecidos, com os instrumentos de avaliação aplicados individualmente por apenas um dos autores (J.O.), em sala reservada no ambulatório ou na enfermaria, quando a criança se encontrava internada.

Os instrumentos de avaliação da sintomatologia e dos transtornos depressivos foram a Escala de Avaliação de Depressão em Crianças - Revisada (CDRS-R) e o Manual Diagnóstico e Estatístico de Desordens Mentais - $4^{a}$ ed. - Texto Revisado (DSM-IV-TR). Após a assinatura do termo de consentimento pelos pais ou responsáveis, os mesmos foram entrevistados para o preenchimento do formulário de pesquisa, realização da anamnese e aplicação da CDRS-R. Numa segunda etapa, uma entrevista individual com a criança foi realizada, na qual o exame mental e a aplicação da CDRS-R foram realizados pelo examinador (J.O.). A avaliação para o diagnóstico dos transtornos depressivos foi feita com base na entrevista com a criança e o responsável, no resultado da CDRS-R, na avaliação dos critérios diagnósticos pelo DSMIV-TR e no exame mental do paciente.

\section{Instrumentos utilizados para avaliar transtorno e sintomatologia depressiva}

\section{Escala para Avaliação de Depressão Infantil - Revisada (CDRS-R) ${ }^{17}$}

A CDRS-R é uma escala amplamente utilizada e conhecida na literatura, com suas propriedades psicométricas consolidadas em pesquisas referentes a transtornos depressivos em crianças e adolescentes ${ }^{3,18}$ e já utilizada em crianças brasileiras $^{19}$. Trata-se de uma entrevista semiestruturada na qual todos os seus itens refletem critérios do DSM-III-R para depressão maior e distimia.

\section{Manual Diagnóstico e Estatístico de Transtornos Mentais - Texto Revisado (DSM-IV-TR) ${ }^{20}$}

Instrumento diagnóstico que consiste numa entrevista clínica para a classificação diagnóstica. Foram empregados os critérios diagnósticos referentes a:

- Episódio Depressivo Maior

- Embora não seja uma entidade diagnóstica distinta, possui critérios diagnósticos bem definidos e serve como base para os diagnósticos dos transtornos de humor.

- Transtorno Distímico

\section{Processamento dos dados e análise estatística}

Os dados foram digitados em um banco de dados específico criado no programa estatístico Epi-info para Windows, versão 3.2.

Para avaliar a associação entre as variáveis categóricas foi utilizado o teste do qui-quadrado de Pearson e, quando os critérios para a utilização deste não eram satisfeitos, o teste exato de Fisher. Na comparação de médias entre os grupos foi utilizado o teste t de Student. Em todos os testes, foi adotado um erro $\alpha$ de 0,05, para a rejeição da hipótese nula.

\section{RESULTADOS}

Esta pesquisa iniciou-se com 31 pacientes portadores de leucemia linfoide aguda (LLA) e 28 portadores de insuficiência renal crônica terminal (IRCT), entretanto, 7 deles (4 com LLA e 3 com IRCT) foram descontinuados, em decorrência do término do tratamento para a doença de base ou por ocorrência de óbito. Assim, participaram efetivamente do estudo 52 crianças e adolescentes, sendo 28 do sexo masculino e 24 do sexo feminino.

A idade média foi de 12,3 (DP = 2,0) anos, sendo a do grupo IRCT 12,7 (DP = 1,9) e a do grupo LLA 11,8 (DP =2,0) anos.

Em relação ao diagnóstico dos transtornos depressivos, realizado através das entrevistas com as crianças e seus responsáveis com base nos critérios do DSM-IV-TR, 3 (5,8\%) casos preenchiam os critérios para episódio depressivo maior, sendo 2 portadores de IRCT e 1 portador de LLA (Tabela 1). Por outro lado, foram encontrados $8(15,4 \%)$ casos com transtorno distímico, todos eles apresentando IRCT (Tabela 1).

Em relação ao diagnóstico de episódio depressivo maior não houve diferença estatística entre as doenças de base, mas quanto ao transtorno distímico houver diferença entre as doenças de base ( $p=0,003)$.

Em relação à faixa etária, não se observou associação com o diagnóstico de episódio depressivo maior ( $p=0,327$ ). Entretanto, houve associação entre o transtorno distímico e a faixa etária ( $p=0,014$ ) (Tabela 2).

Quanto ao tempo de evolução da doença de base, observou-se que a maioria dos pacientes com tempo de evolução de doença de base $\leq 24$ meses $(71,4 \%)$ era portadora de LLA, enquanto que os pacientes com tempo de evolução de doença de base $>24$ meses eram os portadores de IRCT (79,2\%).

Não foi observada diferença estatística na incidência dos transtornos depressivos associada com a evolução da doença de base ( $p=0,559$ para EDM e 0,081 para TD) (Tabela 3). 
Tabela 1. Distribuição dos pacientes de acordo com a doença de base e o transtorno depressivo

\begin{tabular}{|c|c|c|c|c|c|c|c|c|c|c|c|c|}
\hline \multirow[t]{4}{*}{ Doença de base } & \multicolumn{12}{|c|}{ Transtorno depressivo } \\
\hline & \multicolumn{6}{|c|}{ Episódio Depressivo Maior } & \multicolumn{6}{|c|}{ Transtorno Distímico } \\
\hline & \multicolumn{2}{|c|}{ Sim } & \multicolumn{2}{|c|}{ Não } & \multicolumn{2}{|c|}{ Total } & \multicolumn{2}{|c|}{ Sim } & \multicolumn{2}{|c|}{ Não } & \multicolumn{2}{|c|}{ Total } \\
\hline & $\mathrm{n}$ & $\%$ & $\mathrm{n}$ & $\%$ & $\mathrm{n}$ & $\%$ & n & $\%$ & n & $\%$ & $n$ & $\%$ \\
\hline IRCT & 2 & 7,4 & 25 & 92,6 & 27 & 100,0 & 8 & 29,6 & 19 & 70,4 & 27 & 100,0 \\
\hline LLA & 1 & 4,0 & 24 & 96,0 & 25 & 100,0 & 0 & 0,0 & 25 & 100,0 & 25 & 100,0 \\
\hline Total & 3 & 5,8 & 49 & 94,2 & 52 & 100,0 & 8 & 15,4 & 44 & 84,6 & 52 & 100,0 \\
\hline${ }^{*} p$ & \multicolumn{4}{|c|}{0,529} & \multicolumn{8}{|c|}{0,003} \\
\hline
\end{tabular}

* Teste exato de Fisher

Tabela 2. Distribuição dos pacientes conforme faixa etária e transtorno depressivo

\begin{tabular}{|c|c|c|c|c|c|c|c|c|c|c|c|c|}
\hline \multirow[t]{4}{*}{ Faixa etária } & \multicolumn{12}{|c|}{ Transtorno depressivo } \\
\hline & \multicolumn{6}{|c|}{ Episódio Depressivo Maior } & \multicolumn{6}{|c|}{ Transtorno Distímico } \\
\hline & \multicolumn{2}{|c|}{ Sim } & \multicolumn{2}{|c|}{ Não } & \multicolumn{2}{|c|}{ Total } & \multicolumn{2}{|c|}{ Sim } & \multicolumn{2}{|c|}{ Não } & \multicolumn{2}{|c|}{ Total } \\
\hline & $\mathrm{n}$ & $\%$ & $\mathrm{n}$ & $\%$ & $\mathrm{n}$ & $\%$ & $\mathrm{n}$ & $\%$ & $\mathrm{n}$ & $\%$ & $\mathrm{n}$ & $\%$ \\
\hline 8-11 anos & 2 & 10,0 & 18 & 90,0 & 20 & 100,0 & 0 & 0,0 & 20 & 0,0 & 20 & 100,0 \\
\hline $12-15$ anos & 1 & 3,1 & 31 & 96,9 & 32 & 100,0 & 8 & 25,0 & 24 & 75,0 & 32 & 100,0 \\
\hline Total & 3 & 5,8 & 49 & 94,2 & 52 & 100,0 & 8 & 15,4 & 44 & 84,6 & 52 & 100,0 \\
\hline${ }^{*} p$ & \multicolumn{4}{|c|}{0,327} & \multicolumn{8}{|c|}{0,014} \\
\hline
\end{tabular}

*Teste exato de Fisher

Tabela 3. Relação entre o diagnóstico do transtorno depressivo e o tempo de evolução da doença de base

\begin{tabular}{|c|c|c|c|c|c|c|c|}
\hline \multirow[t]{3}{*}{ Transtorno depressivo } & \multicolumn{4}{|c|}{$\begin{array}{l}\text { Tempo de evolução } \\
\text { da doença (meses) }\end{array}$} & \multicolumn{2}{|c|}{ Total } & \multirow[t]{3}{*}{${ }^{*} p$} \\
\hline & \multicolumn{2}{|c|}{$\leq 24$} & \multicolumn{2}{|c|}{$>24$} & \multirow[b]{2}{*}{$\mathrm{n}$} & \multirow[b]{2}{*}{$\%$} & \\
\hline & $\mathrm{n}$ & $\%$ & $\mathrm{n}$ & $\%$ & & & \\
\hline \multicolumn{8}{|c|}{ Episódio depressivo maior } \\
\hline Não & 26 & 92,9 & 23 & 95,8 & 49 & 94,2 & \\
\hline Sim & 2 & 7,1 & 1 & 4,2 & 3 & 5,8 & 0,559 \\
\hline Total & 28 & 100,0 & 24 & 100,0 & 52 & 100,0 & \\
\hline \multicolumn{8}{|l|}{ Transtorno distímico } \\
\hline Não & 26 & 92,9 & 18 & 75,0 & 44 & 84,6 & \\
\hline Sim & 2 & 7,1 & 6 & 25,0 & 8 & 15,4 & 0,081 \\
\hline Total & 28 & 100,0 & 24 & 100,0 & 52 & 100,0 & \\
\hline
\end{tabular}

*Teste exato de Fisher

\section{DISCUSSÃO}

No presente estudo, apesar de os pacientes terem uma doença crônica de base, a taxa de episódio depressivo maior foi de $5,8 \%$, portanto dentro da faixa encontrada na literatura para escolares saudáveis, que indica taxas de transtornos depressivos variando de $0,4 \%$ a $3,0 \%$ em crianças e de 3,3\% a 12,4\% em adolescentes, conforme diferentes regiões do mundo, inclusive o Brasil|21-23. Particularmente em relação aos estudos na população brasileira, nos quais foi avaliada a sintomatologia depressiva, os dados do presente estudo foram menores do que em estudos com escolares saudáveis $22,24,25$. Isso poderia ser explicado porque a maioria dos estudos populacionais tem avaliado apenas sintomatologia depressiva, enquanto que nesta investigação, além da sintomatologia depressiva, foi avaliado o diagnóstico do transtorno depressivo. De fato, isso poderia justificar os nossos resultados, pois os instrumentos para avaliação apenas da sintomatologia depressiva são menos específicos, de aplicação relativamente fácil e, além do mais, a presença de sintomatologia depressiva não implica necessariamente no diagnóstico do transtorno depressivo. Enquanto que o diagnóstico do transtorno depressivo é mais específico, necessita de uma avaliação mais rigorosa, sendo estabelecido segundo os critérios dos manuais diagnósticos.

Em relação ao diagnóstico de transtorno distímico, no presente estudo os pacientes tiveram uma taxa de 15,4\%, que está acima da encontrada na literatura para escolares saudáveis, na qual sua prevalência varia de $0,6 \%$ a 1,7\% para crianças e de $1,6 \%$ a $8 \%$ para adolescentes ${ }^{21,26}$. No que diz respeito às doenças de base estudadas, não foi observada diferença em relação ao diagnóstico de episódio depressivo maior, mas, em relação ao transtorno distímico, sim. Chama a atenção o fato de que todos aqueles com esse diagnóstico psiquiátrico eram pacientes com IRCT, uma doença geralmente mais prolongada que a $\mathrm{LLA}$ e, sendo o transtorno distímico uma condição crônica, que necessita de pelo menos 1 ano da presença da sintomatologia para ser diagnós- 
ticada em crianças e adolescentes, supõe-se que, quanto mais tempo o paciente estiver exposto à doença de base, maior deverá ser o risco de desenvolvê-lo. Assim como no nosso estudo, nenhum caso de transtorno distímico foi encontrado por outros autores quando estudaram apenas crianças portadoras de LLA ${ }^{27}$.

Diferentemente da literatura, não houve diferença tanto na associação entre faixa etária e sintomatologia depressiva, quanto na associação entre faixa etária e episódio depressivo maior ${ }^{5,12,20}$. Por outro lado, verificou-se, com relação ao diagnóstico do transtorno distímico, que todos os pacientes com esse transtorno tinham 12 anos ou mais de idade, corroborando assim os poucos dados existentes na literatura, os quais apontam para o fato de o risco de desenvolvimento desse transtorno ser maior na adolescência ${ }^{18,21,28}$.

Foi observado que a maioria dos pacientes com tempo de evolução até 24 meses tinha LLA, enquanto a maioria daqueles com tempo de evolução de doença acima de 24 meses tinha IRCT. Não foram observadas diferenças no diagnóstico tanto de episódio depressivo maior quanto de transtorno distímico conforme o tempo de evolução da doença de base. Em relação a esse achado, foi observada uma tendência a quanto maior o tempo de evolução da doença, maior o número de sintomas depressivos, provavelmente refletindo a percepção de um estado patológico crônico, pelo tempo que esses sujeitos ficam expostos à doença de base (mais do que 24 meses), ficando, assim, também mais expostos às mudanças impostas por ela $a^{13,29,30}$.

\section{CONCLUSÃO}

Em resumo, a frequência de EDM nessa população corresponde àquela descrita na literatura referente a escolares saudáveis, mas isso não parece ocorrer para o TD, que se apresenta em maior proporção. A ocorrência de EDM parece não se relacionar com a faixa etária, mas o TD parece prevalecer na faixa etária maior, corroborando assim a literatura.

\section{REFERÊNCIAS}

1. Kashani J, Hakami N. Depression in children and adolescents with malignancy. Can J Psychiatry. 1982;27(6):474-7.

2. Rait DS, Jacobsen PB, Lederberg MS, Holland JC. Characteristics of psychiatric consultations in a pediatric cancer center. Am J Psychiatry. 1988;145:363-4.

3. Domènech E, Polaino-Lorente A. Epidemiología de la depresión infantil. Barcelona: Expax; 1990. p.109-30.

4. Kaplan LS, Busner J, Weinhold C, Leon P. Depressive symptoms in children and adolescents with cancer: a longitudinal study. J Am Acad Child Adolesc Psychiatry. 1987;26(5):782-7.

5. Worchel FF, Nolan BF, Wilson VL, Purser JS, Copeland DR, Pfefferbaum B. Assessment of depression in children with cancer. J Pediatr Psychol. 1988;13(1):101-12.
6. Gizynski M, Shapiro VB. Depression and childhood illness. Child Adolesc Soc Work J. 1990;7(3):179-97.

7. Phipps S, Srivasta DK. Repressive adaptation in children with cancer. J Health Psychol. 1997;16(6):521-8

8. Noll RB, Gartstein MA, Vannatta K, Correll J, Bukowski WM, Hobart D. Social, emotional and behavioral functioning of children with cancer. Pediatrics. 1999;103:71-8.

9. Dunitz M, Scheer PJ, Wurst L, Grientschnigg G, Urban E. Depression in children with cancer. Padiatr Padol. 1991;26(6):267-70.

10. Armentia A, Polaino-Lorente A. El impacto del niño con cáncer en el funcionamiento familiar. In: Polaino-Lorente A, Alamo MA, Cano PM, Armentia A, editores. El médico por la família del enfermo? El impacto del enfermo en la familia. Madrid: Ediciones Rialp; 2000. p.33-42.

11. Kovács MJ. A criança e a morte. Tratado de psiquiatria da infância e adolescência. São Paulo: Atheneu, 2003. p.591-8.

12. Soliday E, Kool E, Lande MB. Psychosocial adjustment in children with kidney disease. J Pediatr Psychol. 2000;25(2):93-103.

13. Vieira MA, Lima RAG. Crianças e adolescentes com doença crônica: convivendo com mudanças. Rev Latino-am Enfermagem. 2002;10(4):552-60.

14. Pless I, Nolan T. Risks on maladjustment associated with chronic illness in childhood. In: Shaffer D, Philips I, editores. Prevention of mental disorders, alcohol and other drug. Use in children and adolescents. Washington: US Department of Health and Human Services: 1989, p.78-94

15. Gortmaker SL, Walker DK, Weitzman M, Sobol AM. Chronic conditions, socioeconomic risks, and behavioral problems in children and adolescents. Pediatrics. 1990;85:267-76.

16. Teng CT, Humes EC, Demétrio FN. Depressão e comorbidades clínicas. Rev Psi Clin. 2005:32(3):149-59.

17. Poznanski E0, Freeman LN, Mokros HB. Children's depression rating scale - Revised. Psychopharmacol Bull. 1985;21(4):979-89.

18. Polaino-Lorente A, García Villamisar DA. La depresión infantil en Madrid: un estudio epidemiológico. Madrid: Editorial AC; 1993.

19. Barbosa GA, Dias RD, Gaião AA, Di Lorenzo WF. Escala para avaliação de depressão em crianças - Revisada (CDRS-R): uma análise exploratória. Infanto. 1997;5(1):15-8.

20. American Psychiatric Association. Manual diagnóstico e estatístico de transtornos mentais. $4^{a}$ ed. rev. (DSM-IV-TR). Porto Alegre: Artmed; 2002.

21. Versiani M, Reis R, Figueira I. Diagnóstico do transtorno depressivo na infância e adolescência. J Bras Psiquiatr. 2000; 49(10-12):367-82

22. Bahls SC. Epidemiology of depressive symptoms in adolescents of a public school in Curitiba, Brazil. Rev Bras Psiquiatr. 2002;24(2):63-7.

23. Adewuya A0, Ologum YA. Factors associated with depressive symptoms in Nigeriam adolescents. J Adolesc Health. 2006;39:105-10.

24. Fonseca MHG, Ferreira RA, Fonseca SG. Prevalência de sintomas depressivos em escolares. Pediatria. 2005;27(4):223-32.

25. Barbosa GA, Dias MR, Gaião AA, Di Lorenzo WCG. Depressão infantil: um estudo de prevalência com CDI. Infanto. 1996:4(3):36-40.

26. Masi G, Favilla L, Mucci M, Poli P, Romano R. Depressive symptoms in children and adolescents with dysthymic disorder. Psychopathology. 2001;34:29-35.

27. Bandim JM. Depressão em crianças com leucemia linfoide aguda [tese de doutorado]. Espanha: Universidade Autônoma de Barcelona (UAB); 2000.

28. Canals J, Marti-Henneberg C, Fernandez-Ballart J, Domenech E. A longitudinal study of depression in an urban Spanish pubertal population. Eur Child Adolesc Psychiatry. 1995;4(2):102-11.

29. Castro EK, Piccinini CA. Implicações da doença orgânica crônica na infância para as relações familiares: algumas questões teóricas. Psicologia: reflexão e críticas. 2002;15(3):625-635.

30. Martins LM, França APD, Kimura, M. Qualidade de vida de pessoas com doença crônica. Rev Latino-am Enfermagem. 1996;4(3):5-18.

31. Teng CT, Humes EC, Demetrio FN. Depressão e comorbidades clínicas. Rev. Psiq Clín. 2005;32(3):149-159. 